\title{
The effect of train duration of rewarding stimulation on food self-deprivation
}

\author{
ROBERT A. FRANK, RANDOLPH L. PRESHAW, and ROBERT M. STUTZ \\ University of Cincinnati, Cincinnati, Ohio
}

\begin{abstract}
In order to assess the effect of changes in brain-stimulation reward (BSR) on food intake, train duration of BSR was manipulated during food/BSR competition periods. Train duration, rather than intensity, was used in order to hold the area of stimulation about the electrode tip constant. Train duration had a significant effect on food intake, with longer durations decreasing intake. Increases in food deprivation increased feeding during food/BSR competition, and the tendency for subjects to self-deprive was reduced over the 4 weeks of successive competition testing. Further analyses of the data revealed that stimulation on-time (defined as the product of leverpresses and train duration) was a better predictor of food intake than was leverpressing rate. The results are interpreted as supporting the hypothesis that choices for food or BSR during competition reflect the relative reward value of the alternatives.
\end{abstract}

The potency of brain-stimulation reward (BSR) has been dramatically demonstrated by its ability to compete with behavior that has biologically relevant consequences (Carlisle \& Snyder, 1970; Eckert \& Lewis, 1967; Morgan \& Mogenson, 1966; Rossi \& Stutz, 1978; Routtenberg \& Lindy, 1965; Spies, 1965; Stutz, Rossi, \& Bowring, 1971). It has been argued that self-stimulation competes with other reinforcers by virtue of its rewarding properties (Rossi \& Stutz, 1978; Stutz, Rossi, \& Bowring, 1971) as opposed to its ability to reduce the competitive drive (Spies, 1965). This conclusion is based on the findings that animals who self-starve in order to obtain BSR also self-dehydrate, and that experimenter-administered brain stimulation does not significantly reduce food intake in food-deprived animals who have previously self-starved. Further support for this position is found in research showing that the ability of BSR to compete with sweetened water is a function of the liquid's palatability (Phillips, Morgen, \& Mogenson, 1971) and that food deprivation increases responses for food during BSR/food competition periods (Miliaressis \& Cardo, 1973; Morgan \& Mogenson, 1966). In addition, as demonstrated by Morgan and Mogenson (1966), the intensity of electrical stimulation (which has been shown to be an excellent predictor of BSR magnitude; Hodos \& Valenstein, 1962; Valenstein \& Beer, 1962) greatly influences the responses made for both brain stimulation and water when the two rewards are simultaneously available.

This research was supported by a University of Cincinnati Research Council grant to R. M. Stutz. We gratefully acknowledge the help of Ms. D. Frank in the preparation of this manuscript. Reprint requests should be addressed to R. A. Frank, Department of Psychology, Mail Location 376, University of Cincinnati, Cincinnati, Ohio 45221.
The present study investigated the effect of brainstimulation train duration on the amount of food eaten during limited-access to food and BSR. Train duration has been shown to affect the magnitude of BSR, with longer trains (up to approximately $2 \mathrm{sec}$ ) producing greater rewarding effects (Coleman \& Berard, 1981; Coleman \& Berger, 1978; Deutsch, Chisholm, \& Mason, 1980; Deutsch, Roll, \& Wetter, 1976; Huston, Mills, \& Huston, 1972; Keesey, 1964; Ridgway, Pliskoff, \& McIntire, 1965). Increases in BSR induced by longer train durations are apparently mediated through temporal summation within a discrete population of neurons, while changes in the intensity of the stimulation modify BSR via spatial summation (Gallistel, 1973). Therefore, in order to assess the effect of changing BSR on self-deprivation with the area of stimulation held constant, train duration rather than intensity was manipulated. It was assumed that longer durations more effectively stimulate a finite population of neurons around the electrode tip whereas manipulation of intensity widens the field of stimulation. If the amount of food eaten during simultaneous access to BSR and food is an index of the comparative reward values of the reinforcers, food intake should decrease with increasing train durations.

\section{METHOD}

\begin{abstract}
Subjects
Twenty naive male Sprague-Dawley rats (Blue Spruce Farm, Altamont, New York), weighing $350-425 \mathrm{~g}$, served as subjects. Ambient illumination followed a 12-h light/dark cycle. The subjects had continuous access to water and food (Ralston-Purina Lab Chow) except during periods of food deprivation between testing sessions.
\end{abstract}

\section{Surgery}

The subjects were implanted with bipolar stimulation electrodes 
(Plastic Products Co., electrode diameter $=.25 \mathrm{~mm}$ ) while anesthetized with sodium pentobarbital $(50 \mathrm{mg} / \mathrm{kg}$ ip). Electrodes were aimed at the posterior hypothalamus/medial forebrain bundle (PH/MFB) using the following coordinates: $4.5 \mathrm{~mm}$ posterior to bregma, $1.5 \mathrm{~mm}$ lateral from the midline, and $8.5 \mathrm{~mm}$ below the skull surface, with the skull flat between bregma and lambda.

\section{Apparatus}

All testing was done in six wood and Plexiglas chambers, which contained a metal lever with a microswitch (Scientific Prototype) mounted approximately $5.0 \mathrm{~cm}$ from a floor constructed of aluminum rods spaced about $1.0 \mathrm{~cm}$ apart. The chamber measured $25 \times 22 \times 34 \mathrm{~cm}$. Brain stimulation consisted of constant-current $60-\mathrm{Hz}$ sine waves, and train durations were timed by Hunter interval timers. All subjects were stimulated at $35 \mu \mathrm{A}$ throughout the experiment. Mercury swivel commutators (Scientific Prototype) and bipolar electrode leads (Plastic Products Co.) allowed the animal to be connected to the stimulation circuit. A food cup measuring $5 \times 4 \times 3 \mathrm{~cm}$ was anchored in the rear of each chamber and was filled with $30200-\mathrm{mg}$ Noyes pellets at the start of each BSR/food competition period. Electromechanical counters were used to record leverpresses for BSR.

\section{Procedure}

Subjects were tested for self-stimulation after a 2-week postoperative recovery period. Only animals who consistently responded at least $\mathbf{4 0}$ times per minute (on a CRF schedule) were retained for further testing. Twelve subjects met this criterion. The subjects were then trained to obtain BSR on an FR4 schedule with train durations set at $300 \mathrm{msec}$. This partial reinforcement schedule was used in order to separate the successive trains of BSR. Beyra (1972) has shown that animals pressing for BSR synchronize their presses with stimulation offset. It was felt that separating trains of stimulation with a relatively constant period of no stimulation would accentuate differences among the train durations.

Following the self-stimulation screening and test sessions, baseline feeding sessions began. Food intake was measured in the testing chambers following $0,24,48$, and $72 \mathrm{~h}$ of food deprivation. The testing periods were 45 min long, and during the baseline tests, the BSR lever was not available. During the baseline period, food was available only in the test chamber. The animals were weighed after each test. Following the baseline feeding sessions, the subjects were given 4 days of free feeding in their home cages.

BSR/food competition tests followed the same pattern used during the baseline period, except that the BSR lever and food were available simultaneously. The four train durations used in these competition sessions were $250,500,750$, and 1,000 msec. One-half of the subjects received an ascending order (250-1,000 $\mathrm{msec})$, while the other half experienced a descending order $(1,000$ $250 \mathrm{msec}$ ). Each subject was tested with each train duration at 0 , 24,48 , and $72 \mathrm{~h}$ of food deprivation, with 4 days of free feeding intervening between the tests of each duration. Thus, a given duration was used for 4 consecutive days. Five weeks were required to run the subjects through the four train durations plus the baseline test. The number of food pellets consumed, leverpresses for BSR, and the weight of the animal after each session were recorded.

\section{Histology}

At the completion of the experiment, the subjects were sacrificed with an overdose of sodium pentobarbital and perfused through the heart with a $10 \%$ formal-saline solutions. Brains were subsequently sectioned via the frozen method to determine the locations of the electrode tips.

\section{RESULTS}

CRF leverpressing ranged from 51 to 111 leverpresses/min during the last two self-stimulation screen- ing tests. All animals quickly learned the FR4 schedule and were emitting 53 to 160 presses/min by the last training day. Leverpressing over the competition periods averaged 122.5 presses/min with a range of 63 to $170 \mathrm{presses} / \mathrm{min}$. Average pellets eaten varied from .5 to 25.5 pellets $/ 45$ min session. The 4 days of free feeding which followed the testing series for each train duration were sufficient to allow the animals' weights to return to baseline before testing with the next train duration began (mean of Week $2=357 \mathrm{~g}$; mean of Week $5=362 \mathrm{~g}$ ).

A one-between (counterbalanced train duration groups), two-within (train duration and hour of deprivation) ANOVA was performed on the number of food pellets eaten during the baseline and competition periods. The analysis revealed a significant effect for train duration $[F(1,10)=10.15, p<.05$, conservative $\mathrm{df}]$ and hour of deprivation $[\mathrm{F}(1,10)=29.6$, $\mathrm{p}<.001$, conservative df].

It should be noted that the week of the study also had a significant effect on pellets eaten $[F(1,10)=$ $7.09, \mathrm{p}<.05$, conservative $\mathrm{df}]$. The mean pellets eaten during the baseline week was 20.7. The means for Week 2 through Week 5 were 10.8, 10.3, 13.5, and 17.8 pellets. This weeks effect made it impossible to consider the effect of train duration on any particular subject, since the train duration and weeks effects are confounded for any one subject. This undoubtedly reduced the size of the $F$ ratio observed for train duration. None of the remaining main effects or interactions had a significant effect on pellets eaten. The joint effect of train duration and hour of deprivation on pellets eaten is shown in Figure 1. A one-between, two-within ANOVA was also performed on leverpresses recorded during the competi-

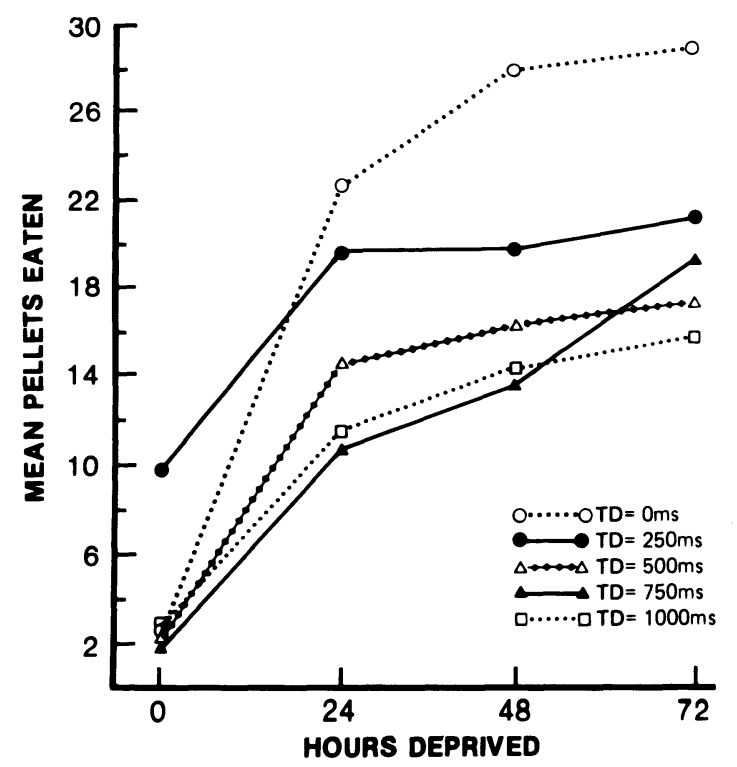

Figure 1. Mean pellets eaten as a function of hours of deprivation and train duration. 


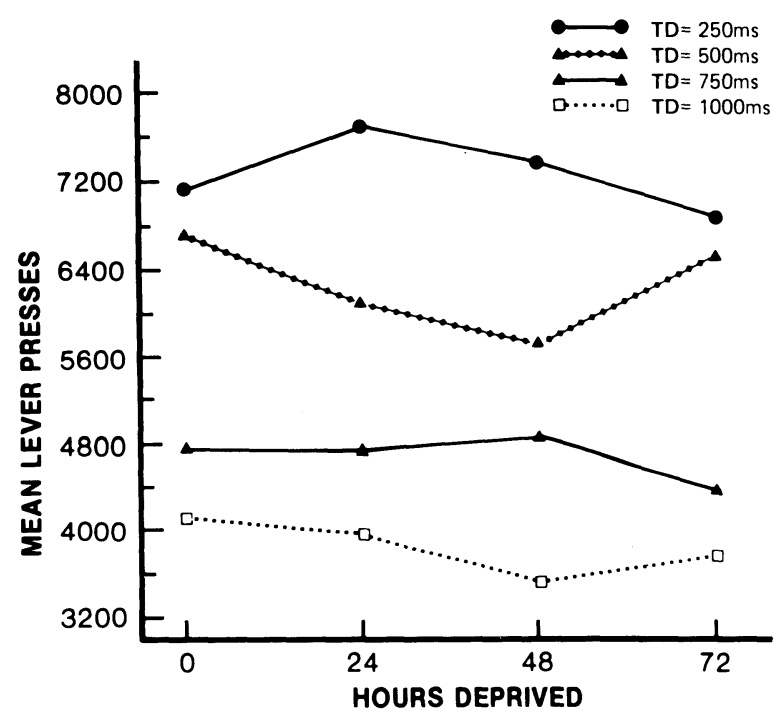

Figure 2. Mean leverpresses as a function of hours of deprivation and train duration.

tion periods. The only significant effect observed was for train duration $[F(1,10)=9.59, p<.05$, conservative df]. The joint effects of train duration and hour of deprivation on leverpressing are shown in Figure 2.

The relationship between the degree of selfdeprivation (as measured by pellets eaten) and leverpressing was examined. The correlation between average leverpressing rate and average pellets eaten during the competition sessions was nonsignificant $(r=$ $-.06)$. However, when those two variables were correlated within animals and across train durations, the more linear relationship seen in Figure 3A was observed. Notice that as leverpresses for BSR increased, pellets eaten also increased. This seemingly paradoxical result is clarified by considering two observations: (1) as train duration increased, pellets eaten decreased, and (2) leverpressing decreased with increasing train duration (as shown in Figure 2). Another potential predictor of self-deprivation was stimulation on-time, defined as the product of number of reinforcers delivered for a particular train duration of BSR times the train duration. Figure 3B plots pellets eaten against stimulation on-time. As can be seen, stimulation on-time is a better linear predictor of self-deprivation than is leverpressing rate.

Histological examination showed the electrode tips to be located in or bordering on the medial forebrain bundle (MFB) at the level of the posterior hypothalamus or in the substantia nigra (both pars compacta and reticulata). The histology of two animals was lost due to transportation of the animals to another laboratory. Self-deprivation appeared to be most pronounced in those subjects having electrodes in the MFB. However, if the electrode was located in the MFB, no particular portion of this structure (e.g., ventromedial or dorsolateral) seemed to be superior for producing self-deprivation. Similar statements could be made about self-stimulation rates. No other effects of electrode locus were noted.

\section{DISCUSSION}

The finding that the train duration of BSR influenced the number of pellets eaten during limitedaccess BSR/food competition periods provides support for the hypothesis that BSR reduces food intake by virtue of its rewarding properties. While stimulationproduced effects unrelated to reward (e.g., failure of sensorimotor integration, amnesia, hunger reduc-

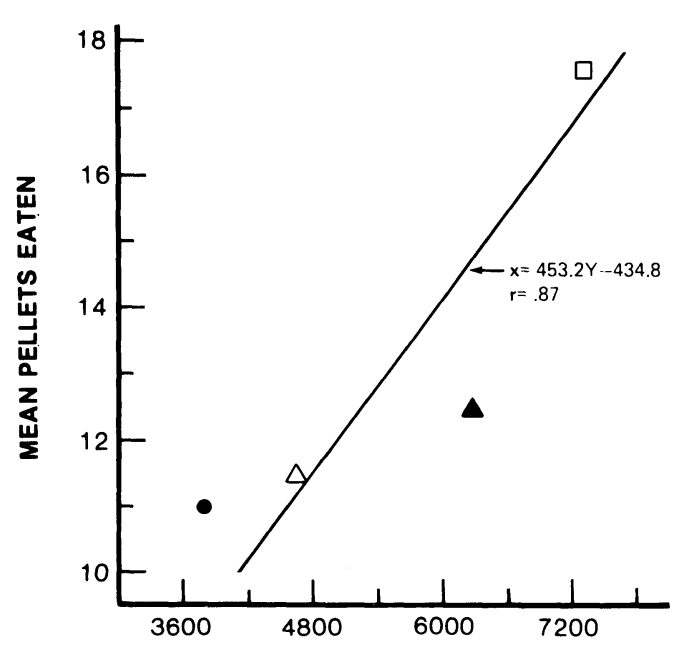

A

MEAN LEVER PRESSING RATE (PER 45 MIN)

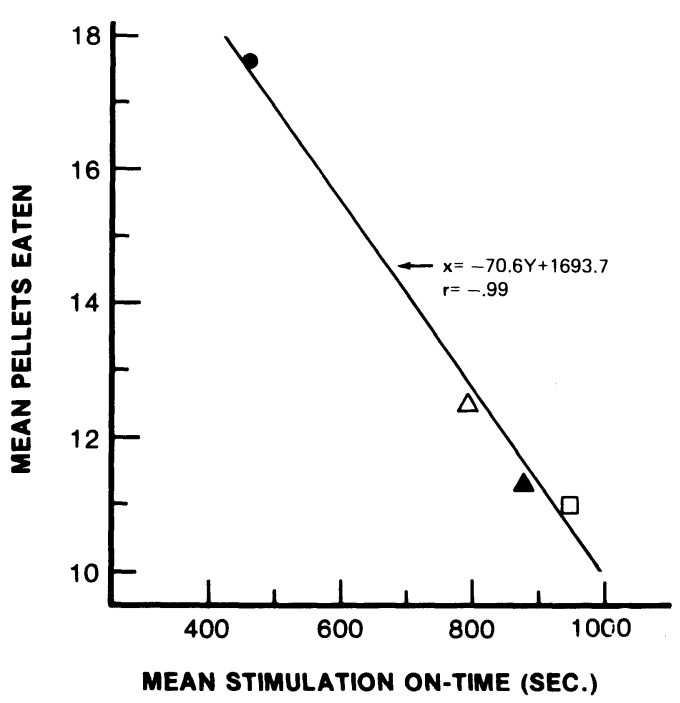

Figure 3. (A) Relationship between mean pellets eaten and mean leverpressing rate. (B) Relationship between mean pellets eaten and mean stimulation on-time. Solid lines are the lines of best fit, and the $\mathbf{r}$ refers to a Pearson product-moment correlation calculated between mean pellets eaten and mean leverpresses or mean stimulation on-time. See Figure 1 for symbol key. 
tion, etc.) could be postulated to account for the results obtained in this study, previous work from our laboratory suggests that none of these explanations can account for the self-deprivation phenomenon. Rossi and Stutz (1978) and Stutz et al. (1971) showed that experimenter-administrated BSR (which removes the element of competition from the experimental situation) does not interfere with food or water intake in previously self-depriving rats. The reward hypothesis is further supported by the observation that the degree of food deprivation influenced the amount of food consumed, suggesting that as food became more rewarding, the effectiveness of BSR in reducing food intake was diminished.

In this study, the ability of increases in train duration to reduce feeding appeared to asymptote between 750 and $1,000 \mathrm{msec}$. Other studies (Deutsch, Roll, \& Wetter, 1976; Keesey, 1964) report asymptotic effectiveness of train duration between 1 and $2 \mathrm{sec}$. This difference in asymptotic performance could be due to the relative sensitivity of the measures employed. The finding that the effectiveness of train duration depends on stimulation intensity (Deutsch, Chisholm, \& Mason, 1980) may also account for this difference. It is difficult to assess the importance of this factor, since Deutsch et al. (1976) do not report the intensities they used and Keesey (1964) used squarewave stimulation.

Previous research by Morgan and Mogenson (1966) demonstrated that manipulations of intensity significantly altered the effectiveness of BSR in reducing water intake when BSR and water were simultaneously available. However, it was unclear whether the changes in self-dehydration were due to changes in the field of stimulation (that "pulled in" neurons critical for self-dehydration), increases in BSR, or both. By manipulating the train duration of BSR (and thereby holding the field of stimulation constant), the present investigation has shown that temporal summation within a discrete population of neurons can lead to increases in self-deprivation.

Leverpressing was significantly reduced as train duration increased. This finding was expected, since animals do not leverpress while the stimulation is on (Beyra, 1972). Several studies have reported that a subject's leverpressing rate is a good predictor of self-deprivation (Rossi \& Stutz, 1978; Stutz, Rossi, \& Bowring, 1971). However, in this investigation the leverpressing rate of a subject did not correlate highly with degree of self-deprivation $(r=-.06)$. In addition, a within-subject analysis showed that as leverpresses increased in response to decreasing train durations, the degree of self-deprivation decreased (see Figure 3A). As can be seen in Figure 3B, stimulation on-time was an excellent predictor of the number of pellets eaten. This fact lends further support to the claim that the train duration of BSR was an important factor in determining the amount of feeding that occurred when food and BSR were simultaneously available. One final relationship between leverpressing and feeding that should be noted is that leverpressing remained constant over hours of deprivation while feeding increased significantly. This result indicates that leverpressing and feeding are at least partially independent. On the basis of this evidence, one may infer that subjects had time to eat more without compromising a high rate of selfstimulation, but did not eat until food deprivation had become severe. One possible explanation for this finding is that BSR and food are being compared and, as deprivation increases, food becomes more rewarding while BSR does not change.

The decrease in self-deprivation observed across the weeks of the study has been reported in other studies that have examined multiple BSR/food competition sessions (Santos \& Routtenberg, 1972; Frank, Pritchard, \& Stutz, 1982). Santos and Routtenberg (1972) have interpreted this effect as the result of adaptation to deprivation stress. This explanation seems to apply to the research presented here. In addition, it should be remembered that, since high rates of leverpressing did not preclude substantial bouts of feeding, subjects may be learning to make optimal use of the available time during the competition sessions by alternating between food and BSR. Recent analyses of data collected in our laboratory (unpublished observations) during continuous access to food, a glucose-saccharin solution, and BSR indicate that rats were able to maintain a constant level of food intake despite quadrupling fluid intake (as compared with baseline water intake) and averaging 40 leverpresses/min for BSR for $24 \mathrm{~h}$. This impressive behavioral output began with exclusive pressing for BSR, then alternating responses for BSR and the sweetened water, and finally periods of shuttling between the food, BSR, and sweetened water. Animals may be learning to alternate responses among the available rewards across successive test sessions. This learning process may reflect the gradual expression of an elastic demand for BSR as compared with an inelastic demand for food (Hursh \& Natelson, 1981).

\section{REFERENCES}

Beyra, M. Train length perception during self-stimulation behaviour. Experientia, 1972, 28, 912-913.

Carlisle, H. J., \& Snyder, E. The interaction of hypothalamic self-stimulation and temperature regulation. Experientia (Separatum), 1970, 26, 1092-1093.

Coleman, W. R., \& Berard, D. R. Psychophysical mapping of reward system operating characteristics: Utility transforms of stimulation duration. Physiology \& Behavior, 1981, 26, 61-68.

Coleman, W. R., \& Berger, L. H. Utility scaling of intracranial reinforcement duration. Physiology \& Behavior, 1978, 21, 485-490.

Deutsch, J. A., Chisholm, D., \& Mason, P. A. Adaptation to rewarding brain stimuli of differing amplitude. Behavioral and Neural Biology, 1980, 29, 359-364. 
Deutsch, J. A., Roll, P. L., \& Wetter, F. Choice between rewarding brain stimuli of differing lengths. Behavioral Biology, 1976, 18, 369-377.

Eckert, B., \& Lewis, M. Competition between drives for intracranial stimulation and sodium chloride by adrenalectomized $\mathrm{NaCl}$-deprived rats. Journal of Comparative and Physiological Psychology, 1967, 64, 349-352.

Frank, R. A., Pritchard, W. S., \& Stutz, R. M. Food versus intracranial self-stimulation: Failure of limited-access selfdepriving rats to self-deprive in a continuous access paradigm. Behavioral and Neural Biology, 1982, 33, 503-508.

Gallistel, C. R. Self-stimulation: Reward and motivation. In J. A. Deutsch (Ed.), The physiological basis of memory. New York: Academic Press, 1973.

Hodos, W., \& VAlenstein, E. S. An evaluation of response rate as a measure of rewarding intracranial stimulation. Journal of Comparative and Physiological Psychology, 1962, 55, 80-84.

Hursh, S. R., \& Natelson, B. H. Electrical brain stimulation and food reinforcement dissociated by demand elasticity. Physiology \& Behavior, 1981, 26, 509-515.

Huston, J. P., Mills, A. W., \& Huston, R. Strength-duration function of hypothalamic self-stimulation. Behavioral Biology, 1972, 7, 383-390.

KEESEY, R. E. Duration of stimulation and the reward properties of hypothalamic stimulation. Journal of Comparative and Physiological Psychology, 1964, 58, 201-207.

Miliaressis, E., \& Cardo, B. Self-stimulation versus food reinforcement. Comparative study of two different nervous structures, the lateral hypothalamus and ventral tegmental area of the mesencephalon. Brain Research, 1973, 57, 75-83.

Morgan, C. W., \& Mogenson, G. J. Preference of water-deprived rats for stimulation of the lateral hypothalamus. Psychonomic Science, 1966, 6, 337-338.
Phillips, A. G., Morgan, C. W., \& Mogenson, G. J. Changes in self-stimulation preference as a function of incentive of alternative rewards. Review of Canadian Psychology, 1970, 24, 289-297.

Ridgway, J. E., Pliskoff, S. S., \& McIntire, R. W. The utility of the microcoulomb as a predictor of reinforcement value in electrical self-stimulation of the rat brain. Psychonomic Science, 1965, 3, 109-110.

Rossi, R. R., \& STUtz, R. M. The self-deprivation phenomenon: Competition between appetitive rewards and electrical stimulation of the brain. Physiological Psychology, 1978, 6, 204-208.

ROUTTENBERG, A., \& LindY, J. Effects of availability of rewarding septal and hypothalamic stimulation on bar pressing for food under conditions of deprivation. Journal of Comparative and Physiological Psychology, 1965, 60, 158-168.

Santos, R. M., \& Routtenberg, A. Attenuation of brain stimulation: Adaptation effects. Physiology \& Behavior, 1972, 9, 831-837.

SPIEs, G. Food versus intracranial self-stimulation reinforcement in food deprived rats. Journal of Comparative and Physiological Psychology, 1965, 60, 153-157.

Stutz, R. M., Rossi, R. R., \& Bowring, A. M. Competition between food and rewarding brain shock. Physiology \& Behavior, 1971, 7, 753-757.

VAlenste in, E. S., \& BeER, B. Reinforcing brain stimulation in competition with water reward and shock avoidance. Science, 1962, 137, 1052-1054.

(Manuscript received September 2, 1981; revision accepted for publication December 21, 1981.) 